\title{
MicroRNA-32 silences WWP2 expression to maintain the pluripotency of human amniotic epithelial stem cells and $\beta$ islet-like cell differentiation
}

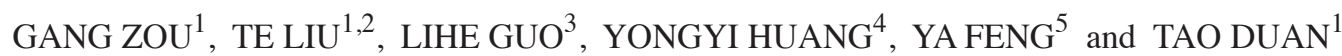 \\ ${ }^{1}$ Department of Obstetrics, Shanghai First Maternity and Infant Hospital, Tongji University School of Medicine, \\ Shanghai 200040; ${ }^{2}$ Shanghai Geriatric Institute of Chinese Medicine, Longhua Hospital, Shanghai University of \\ Traditional Chinese Medicine, Shanghai 200031; ${ }^{3}$ Institute of Biochemistry and Cell Biology, \\ Shanghai Institute for Biological Sciences, Chinese Academy of Sciences, Shanghai 200031, P.R. China; \\ ${ }^{4}$ Laboratoire PROTEE, Batiment R, University du Sud Toulon-Var, 83957 La Garde Cedex, France; \\ ${ }^{5}$ Department of Neurology, Tongji Hospital, Tongji University School of Medicine, Shanghai 200040, P.R. China
}

Received November 17, 2015; Accepted December 20, 2017

DOI: $10.3892 /$ ijmm.2018.3436

\begin{abstract}
Human amniotic epithelial stem cells (HuAECs) exhibit pluripotent characteristics, which are similar to those of embryonic stem cells, and can differentiate into various adult tissues and cells through directed induction. However, in culture, HuAECs tend to lose their pluripotency, and their directed differentiation capability declines with increasing passage number. The stem cell pluripotency factor octamer-binding protein 4 (Oct4) is an important transcription factor that promotes stem cell self-proliferation and maintains their pluripotency. Previous studies have demonstrated that WW domain containing E3 ubiquitin protein ligase 2 (WWP2) negatively regulates Oct 4 expression and stem cell pluripotency. Therefore, the present study aimed to investigate the regulation of WWP2 by microRNAs (miRs), and to evaluate the expression of the downstream factor Oct4 and the maintenance of HuAEC pluripotency. Bioinformatics analysis identified a complementary binding site for miR-32 in the 3'untranslated region of the WWP2 gene, thus suggesting that it may be a target gene of miR-32. Post-infection of HuAECs with a vector overexpressing miR-32, the endogenous expression of WWP2 was significantly decreased, whereas Oct4 expression was
\end{abstract}

Correspondence to: Professor Te Liu, Shanghai Geriatric Institute of Chinese Medicine, Longhua Hospital, Shanghai University of Traditional Chinese Medicine, 365 Xiangyang Road, Shanghai 200031, P.R. China

E-mail: teliu79@126.com

Professor Tao Duan, Department of Obstetrics, Shanghai First Maternity and Infant Hospital, Tongji University School of Medicine, 2699 Gaoke xi Road, Shanghai 200040, P.R. China E-mail: tduan@yahoo.com

Key words: human amniotic epithelial stem cells, microRNA-32, WW domain containing E3 ubiquitin protein ligase 2, pluripotency, $\beta$ islet-like cells significantly increased. Furthermore, miR-32-infected cells differentiated into $\beta$ islet-like cells by directed induction. The results indicated that after induction, HuAECs overexpressing miR-32 also overexpressed the biomarkers of $\beta$ islet-like cells. In addition, the ability to secrete insulin was markedly enhanced in response to glucose stimulation, in cells overexpressing miR-32. In conclusion, the present study suggested that miR-32 may effectively inhibit WWP2 expression in HuAECs and promote Oct4 overexpression to maintain their pluripotency.

\section{Introduction}

Originally derived from trophoblasts, human amniotic epithelial stem cells (HuAECs) are a specific type of cell that possess the pluripotency of embryonic stem cells (ESCs) (1-3). HuAECs can be induced to differentiate into various human tissues and cells, and have specific therapeutic effects in some human diseases (1-3). However, it is difficult to maintain the pluripotency of HuAECs for long periods in culture. Following primary isolation of HuAECs, pluripotency factors, including octamer-binding protein 4 (Oct4), SRY-box 2 (Sox2) and Nanog, are highly expressed; however, by the fifth passage, the expression levels of these factors are decreased to $10 \%$ of the original levels, and after subsequent passages, HuAECs hardly express these factors. Furthermore, at this point, HuAECs can no longer be induced to differentiate into other adult cells, retaining only the phenotype and partial characteristics of epithelial cells (1). These issues have seriously hindered the use of HuAECs.

Ubiquitination is a post-translational modification process by which ubiquitin becomes covalently bound to a substrate protein by enzymatic action (4-8). The function of ubiquitination is to mark target proteins so that they are recognized and degraded by proteasomes (4-8). Comprising 76 amino acids, ubiquitin is a small polypeptide with a conserved sequence, which is present universally and ubiquitously in eukaryotic cells (4-8). The ubiquitination process begins with an E1 ubiquitin-activating enzyme, which, in an ATP-dependent reaction, activates a single free ubiquitin molecule by forming 
a thioester bond between the Cys residue of the activity center of E1 and the C-terminus of ubiquitin. The activated ubiquitin is channeled to an E2 ubiquitin-conjugating enzyme, which, together with the recruited specific substrate protein and an E3 ubiquitin ligase, mediates the transfer of ubiquitin from E2 to the target protein. The recognition and degradation of ubiquitinated proteins by proteasomes is a non-specific process; therefore, the E3 ligase is a crucial factor in the process of protein degradation, determining the specificity of the protein degradation reaction. In most cases, the ubiquitination reaction involves one E1 ubiquitin-activating enzyme, and numerous E2 ubiquitin-conjugating enzymes and E3 ubiquitin ligases. Quantitatively, one E1 interacts with several E2 enzymes, whereas one E2 enzyme interacts with many more E3 ubiquitin ligases. The inverted 'pyramid' structure formed by E3, E2 and E1 is a large and complex signaling cascade network, which extensively and accurately regulates cell activities. The highly specific protein degradation mechanism in the cell is predominantly determined by E3 ubiquitin ligase, and all E3 ubiquitin ligases are capable of linking a target protein to a particular E2 enzyme. There are three major categories of E3 ubiquitin ligase: Those containing a homologous to the E6-AP carboxyl terminus (HECT) domain; those containing a RING domain; and those containing a U-box protein domain. The HECT domain-containing E3 ubiquitin ligases act mainly by forming a thioester bond with ubiquitin, which is essential to catalysis, whereas the RING domain-containing E3 ubiquitin ligases provide the binding sites for E2 enzymes and the substrate protein, so that the E2 enzyme can catalyze direct transfer of ubiquitin to the substrate (4-8). WW domain containing E3 ubiquitin protein ligase 2 (WWP2) (also known as atrophin-1-interacting protein 2) is a novel member of the E3 ubiquitin ligase family, which specifically induces ubiquitination of Oct4, ultimately leading to its degradation (9-14). Following the loss of ligase activity of WWP2 by a point mutation in the active site, the ubiquitination level of Oct4 is significantly reduced (9-14). Conversely, once a ubiquitin molecule is fused to the $\mathrm{N}$-terminus of Oct4, a negative correlation is observed between Oct4 level and cellular ubiquitination level (9-14). In addition, when WWP2 is overexpressed in ESCs, it may inhibit the activity of endogenous Oct4 (9-14).

Based on these findings, the present study aimed to investigate whether microRNA (miR)-32 weakens the ubiquitination of Oct4 by specifically inhibiting WWP2 expression, thus maintaining the pluripotency of HuAECs and enhancing their capacity for differentiation into $\beta$ islet-like cells by directed induction.

\section{Materials and methods}

HuAECs isolation and culture. The present study was approved by the ethics committee of Shanghai Geriatric Institute of Chinese Medicine, Longhua Hospital, Shanghai University of Traditional Chinese Medicine (Shanghai, China). From May 2015 to December 2015, human placentas were obtained with the written informed consent of pregnant women (age, 28 \pm 3 ), who were negative for human immunodeficiency virus-I, hepatitis B and hepatitis $\mathrm{C}$. The institutional ethics committee approved the use of human amnions. Amnion membranes were peeled mechanically from the chorions of placentas obtained from women that underwent an uncomplicated Cesarean section. The epithelial layers, with the basement membrane attached, were obtained and used to harvest HuAECs, as previously described, with some modification (15-17). Briefly, the membrane was placed in a 250-ml flask containing Dulbecco's modified Eagle's medium (DMEM) and was cut into $0.5-1.0 \mathrm{~cm}^{2}$ segments using a razor. The segments were digested with $0.25 \%$ trypsin-EDTA at $37^{\circ} \mathrm{C}$ for $45 \mathrm{~min}$. The resulting cell suspension was seeded in a 6-well plate in DMEM supplemented with $10 \%$ fetal calf serum (GE Healthcare, Chicago, IL, USA), penicillin (100 U/ml) and glutamine $(0.3 \mathrm{mg} / \mathrm{ml})$ at $37^{\circ} \mathrm{C}$ in a humidified tissue culture incubator containing $5 \% \mathrm{CO}_{2}$. Successful infection of mutated miR (miR-Mut) and miR-32 was confirmed by RT-qPCR (Fig. 1).

Induction of HuAECs differentiation into $\beta$ islet-like cells and $C$-peptide assay. According to a previous study (18), to induce their differentiation into insulin-producing $\beta$ islet-like cells, HuAECs $\left(1 \times 10^{6}\right)$ were seeded into a $10-\mathrm{cm}$ dish and cultured in serum-free DMEM containing $25 \mathrm{mM}$ D-glucose, N2 supplement (Gibco; Thermo Fisher Scientific, Inc., Waltham, MA, USA), $1 \%$ antibiotic-antimycotic and $10 \mathrm{mM}$ nicotinamide (Sigma-Aldrich; Merck KGaA, Darmstadt, Germany) for 2 weeks; medium was changed twice a week. Cell culture supernatants were collected at appropriate time points, centrifuged to remove cell debris, and the C-peptide levels were assessed using a human $\mathrm{C}$-peptide radioimmunoassay kit (China Diagnostics Medical Corporation, Beijing, China).

RNA extraction and reverse transcription-quantitative polymerase chain reaction ( $R T-q P C R)$ analysis. Total RNA was isolated from each cell sample using TRIzol ${ }^{\circledR}$ reagent (Invitrogen; Thermo Fisher Scientific, Inc.) according to the manufacturer's protocol. RNA samples were digested with DNase I (Sigma-Aldrich; Merck KGaA), quantified and reverse transcribed into cDNA using a ReverTra Ace- $\alpha$ First Strand cDNA Synthesis kit (Toyobo Life Science, Osaka, Japan). A RealPlex4 real-time PCR detection system (Eppendorf, Hamburg, Germany) was used to conduct RT-qPCR, with SYBR-Green Real-time PCR Master mix (Toyobo Life Science) used as the detection dye. The RT-qPCR amplification conditions were as follows: 1 cycle of initial denaturation at $95^{\circ} \mathrm{C}$ for $2 \mathrm{~min}$; and 40 cycles of denaturation at $95^{\circ} \mathrm{C}$ for $15 \mathrm{sec}$ and annealing at $58^{\circ} \mathrm{C}$ for $45 \mathrm{sec}$ and extension at $72^{\circ} \mathrm{C}$ for $42 \mathrm{sec}$; Then, 1 cycle of final extension at $72^{\circ} \mathrm{C}$ for $10 \mathrm{~min}$. Target cDNA was quantified using the relative quantification method. The quantification cycle $(\mathrm{Cq})$ method was used to determine gene expression relative to a control (calibrator), and steady-state mRNA levels were reported as the fold difference relative to the calibrator. For each sample, the marker gene $\mathrm{Cq}$ values were normalized using the formula $\Delta \mathrm{Cq}=\mathrm{Cq}$ _genes - Cq_18s ribosomal RNA (rRNA). The relative expression levels were determined according to the following formula: $\Delta \Delta \mathrm{Cq}=\Delta \mathrm{Cq}$ _sample group - $\Delta \mathrm{Cq}$ _control group. The $2^{-\Delta \Delta \mathrm{Cq}}$ method was used to calculate the relative expression levels of marker genes (19). The calibrator used was the 18s rRNA gene. The cDNA of each gene was amplified using primers shown in Table I.

Luciferase reporter assay. The luciferase reporter assay was performed according to a previously described method $(15,20)$. NIH-3T3 cells were seeded at 3x10\%/well in 48-well plates and were 
Table I. Polymerase chain reaction primers

\begin{tabular}{|c|c|c|}
\hline Gene name & Primers $\left(5^{\prime} \rightarrow 3^{\prime}\right)$ & Size (bp) \\
\hline Nanog & $\begin{array}{l}\text { F: GATTTGTGGGCCTGAAGAAA } \\
\text { R ATGGAGGAGGGAAGAGGAGA }\end{array}$ & 93 \\
\hline Oct4 & $\begin{array}{l}\text { F: CAGTGCCCGAAACCCACAC } \\
\text { R: GGAGACCCAGCAGCCTCAAA }\end{array}$ & 161 \\
\hline Sox2 & $\begin{array}{l}\text { F: AACCCCAAGATGCACAACTC } \\
\text { R: GCTTAGCCTCGTCGATGAAC }\end{array}$ & 100 \\
\hline Insulin & $\begin{array}{l}\text { F: TTCTACACACCCAAGACCCG } \\
\text { R: CAATGCCACGCTTCTGC }\end{array}$ & 130 \\
\hline Pdx1 & $\begin{array}{l}\text { F: CCTTTCCCATGGATGAAGTC } \\
\text { R: CGTCCGCTTGTTCTCCTC }\end{array}$ & 96 \\
\hline Pax6 & $\begin{array}{l}\text { F: GTTGGTATCCGGGGACTTC } \\
\text { R: TCCGTTGGAACTGATGGAGT }\end{array}$ & 101 \\
\hline Nkx 2.2 & $\begin{array}{l}\text { F: TCTACGACAGCAGCGACAAC } \\
\text { R: GGAGCTTGAGTCCTGAGGG }\end{array}$ & 110 \\
\hline Glut2 & $\begin{array}{l}\text { F: TGTGCCACACTCACACAAGA } \\
\text { R: GACAGTGAAAACCAGGGTCC }\end{array}$ & 96 \\
\hline GCK & $\begin{array}{l}\text { F: GATGGATGTCACAAGGAGCC } \\
\text { R: CCTTCTTCAGGTCCTCCTCC }\end{array}$ & 95 \\
\hline Isl1 & $\begin{array}{l}\text { F: TGTTTGAAATGTGCGGAGTG } \\
\text { R: TCTTGCTGAAGCCGATGC }\end{array}$ & 142 \\
\hline 18S rRNA & $\begin{array}{l}\text { F: CAGCCACCCGAGATTGAGCA } \\
\text { R: TAGTAGCGACGGGCGGTGTG }\end{array}$ & 253 \\
\hline \multicolumn{3}{|c|}{$\begin{array}{l}\text { F, forward; GCK, glucokinase; Glut2, glucose transporter 2; } \\
\text { Nkx2.2, NK2 homeobox 2; Oct4, octamer-binding protein 4; Pax6, } \\
\text { paired box 6; PCR, polymerase chain reaction; Pdx1, pancreatic } \\
\text { duodenal homeobox-1; R, reverse; Sox2, SRY-box 2; Isl1, ISL LIM } \\
\text { homeobox 1; rRNA, ribosomal RNA. }\end{array}$} \\
\hline
\end{tabular}

cotransfected with 400 ng pRNAT-CMV-miR-32, pRNAT-CMV or pRNAT-CMV-miR-32-Mut, and 20 ng pGL-WWP2-3' untranslated region (UTR)-wild-type (WT) or pGL-WWP2 3'UTR-Mut, and pRL-TK (Promega Corporation, Madison, WI, USA) using Lipofectamine $^{\circledR} 2000$ reagent (Invitrogen; Thermo Fisher Scientific, Inc.) according to the manufacturer's protocol. After $48 \mathrm{~h}$ of transfection, luciferase activity was measured using a dual-luciferase reporter assay system (Promega Corporation).

Immunofluorescence staining. Cultured cells were washed three times with fetal calf serum and fixed with 4\% paraformaldehyde (Sigma-Aldrich; Merck KGaA) for $30 \mathrm{~min}$. After blocking, the cells were incubated with primary antibodies (Table II) overnight at $4^{\circ} \mathrm{C}$, and then with goat anti-rabbit IgG H\&L (Alexa Fluor ${ }^{\circledR} 555$ ) pre-adsorbed (1:200; ab150086; Abcam, Cambridge, UK) and $5 \mu \mathrm{g} / \mathrm{ml} 4$ ',6-diamidino-2-phenylindole (Sigma-Aldrich; Merck KGaA) at $37^{\circ} \mathrm{C}$ for $30 \mathrm{~min}$. The cells were then washed thoroughly with Tris-buffered saline-Tween (TBST) [25 mM Tris/ $\mathrm{HCl}(\mathrm{pH} 8.0), 125 \mathrm{mM} \mathrm{NaCl}$ and $0.05 \%$ Tween-20] and viewed under a fluorescence microscope (DMI3000; Leica Microsystems, Inc., Buffalo Grove, IL, USA).
Table II. Antibodies

\begin{tabular}{lll}
\hline Antibody & \multicolumn{1}{c}{ Supplier } & Application \\
\hline Rabbit anti-human & Cell Signaling & IF $(1: 200)$ \\
Oct4 (sc-9081) & Technology, Inc. & WB $(1: 1,000)$ \\
Rabbit anti-human & Cell Signaling & IF $(1: 200)$ \\
Nanog (sc-33759) & Technology, Inc. & WB $(1: 1,000)$ \\
Rabbit anti-human & Santa Cruz & IF $(1: 200)$ \\
Sox2 (sc-20088) & Biotechnology, Inc. & WB $(1: 1,000)$ \\
Mouse anti-human & Santa Cruz & \\
insulin (sc-377071) & Biotechnology, Inc. & WB $(1: 1,000)$ \\
Rabbit anti-human & Santa Cruz & \\
PDX1 (sc-25403) & Biotechnology, Inc. & WB $(1: 1,000)$ \\
Mouse anti-human & Santa Cruz & IF $(1: 200)$ \\
WWP2 (sc-398090) & Biotechnology, Inc. & WB $(1: 1000)$ \\
Rabbit anti-human & Cell Signaling & WB $(1: 1,000)$ \\
GADPH (sc-25778) & Biotechnology, Inc. & \\
\hline
\end{tabular}

Cell Signaling Technology, Inc., Danvers, MA, USA. IF, immunofluorescence; Oct4, octamer-binding protein 4; PDX1, pancreatic duodenal homeobox-1; Sox2, SRY-box 2; WB, western blotting; WWP2, WW domain containing E3 ubiquitin protein ligase 2.

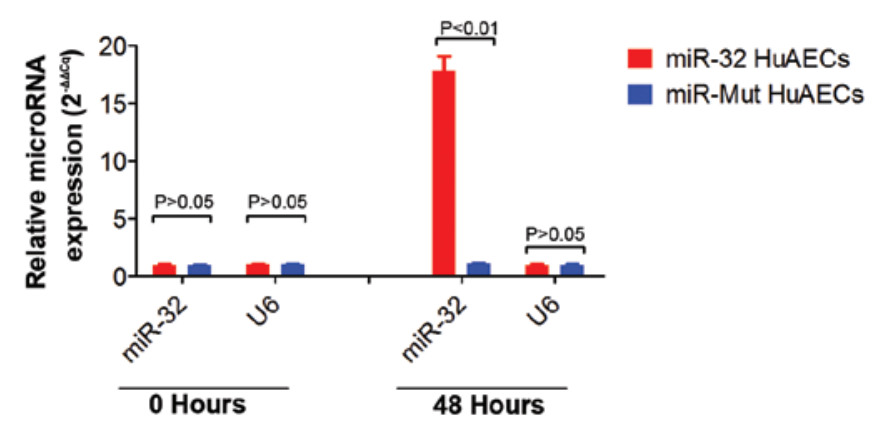

Figure 1. Confirmation of successful infection of HuAECs with miR-32. HuAECs, human amniotic epithelial stem cells; miR-32, microRNA-32.

Western blot analysis. HuAECs were lysed in $2 \mathrm{X}$ loading lysis buffer [50 mM Tris- $\mathrm{HCl}$ ( $\mathrm{pH}$ 6.8), $2 \%$ sodium dodecyl sulfate, $10 \% \beta$-mercaptoethanol, $10 \%$ glycerol and $0.002 \%$ bromophenol blue]. The concentration of total protein was then determined by Enhanced BCA Protein Assay kit (Beyotime Institute of Biotechnology, Shanghai, China). The total proteins $(15 \mu \mathrm{l}, 25 \mu \mathrm{g} / \mu \mathrm{l})$ from cultured cells were then separated by $12 \%$ SDS-PAGE and transferred onto Hybrid-polyvinylidene fluoride membranes (EMD Millipore, Bedford, MA, USA). The membranes were blocked with $5 \%(\mathrm{w} / \mathrm{v})$ non-fat dried milk in TBST and were washed four times with TBST at room temperature (15 min/wash) prior to incubation with primary antibodies (Table II) in 5\% (w/v) non-fat dried milk in TBST. After further washing, the membranes were incubated with horseradish peroxidase-conjugated goat anti-rabbit IgG secondary antibody (1:1,000; sc-2004; Santa Cruz Biotechnology, Dallas, TX, USA) at $37^{\circ} \mathrm{C}$ for $1 \mathrm{~h}$. After washing four times with TBST at room temperature $(15 \mathrm{~min} /$ wash), the immunoreactive protein bands were visualized 
by enhanced chemiluminescence (ECL) using an ECL kit (PerkinElmer, Inc., Waltham, MA, USA).

Northern blot analysis. Northern blotting was performed according to a previously described method (15). For all groups, $20 \mu \mathrm{g}$ good quality total RNA was analyzed on a 7.5 M urea, $12 \%$ polyacrylamide denaturing gel and was transferred to Hybond $\mathrm{N}^{+}$nylon membrane (GE Healthcare). Ultraviolet light, for $30 \mathrm{sec}$ at $1,200 \mathrm{mjoule} / \mathrm{cm}^{2}$, was used to crosslink the membranes. The membranes were hybridized with the miR-32 antisense starfire probe, 5'-ATAACGTGT AATGATTCAACGT-3' (Integrated DNA Technologies, Coralville, IA, USA), to detect the 22-nt miR-32 fragments, according to the manufacturer's protocol. After washing by Northern washing buffer $\left(25 \mathrm{mM} \mathrm{NaH} \mathrm{PO}_{4} \cdot 2 \mathrm{H}_{2} \mathrm{O}, 25 \mathrm{mM}\right.$ $\mathrm{Na}_{2} \mathrm{HPO}_{4} \cdot 12 \mathrm{H}_{2} \mathrm{O}, 1 \mathrm{mM}$ EDTA, $1 \%$ SDS, $5 \%$ formamide deionized), the membranes were exposed to Kodak XAR-5 films for 20-40 h (Sigma-Aldrich; Merck KGaA). All membranes were hybridized with a human U6 snRNA probe as a positive control, 5'-GCAGGGGCCATGCTAATCTTC TCTGTATCG-3'. The exposure times for the U6 control probe varied between 15 and $30 \mathrm{~min}$.

Co-immunoprecipitation (co-IP) assay. Cells were seeded at $3 \times 10^{5} /$ well in 6 -well plates and were cultured until they reached $85 \%$ confluence. The cells were then lysed in $500 \mu \mathrm{l}$ modified cell lysis buffer [20 mM Tris ( $\mathrm{pH} 7.5), 150 \mathrm{mM}$ $\mathrm{NaCl}, 1 \%$ Triton $\mathrm{X}-100,1 \mathrm{mM}$ EDTA, sodium pyrophosphate, $\beta$-glycerophosphate, $\mathrm{Na}_{3} \mathrm{VO}_{4}$ and leupeptin) (Beyotime Institute of Biotechnology) for western blotting and co-IP. After lysis, each sample was clarified by centrifugation to remove insoluble debris and the supernatant was preincubated with $20 \mu \mathrm{g}$ protein A agarose beads (Beyotime Institute of Biotechnology) for $30 \mathrm{~min}$ at $4^{\circ} \mathrm{C}$ with agitation. Subsequently, the samples were centrifuged and transferred to a fresh $1.5 \mathrm{ml}$ tube. The samples were incubated with antibodies (Table II) for $90 \mathrm{~min}$ prior to the addition of $20 \mu \mathrm{g}$ protein A agarose beads to capture immune complexes. The pelleted beads were washed three times with $500 \mu \mathrm{l}$ cell lysis buffer, dissolved in $4 \mathrm{X}$ SDS-PAGE sample loading buffer and heated for $10 \mathrm{~min}$ at $95^{\circ} \mathrm{C}$.

$\mathrm{KCl}$ and tolbutamide stimulation experiments. According to the protocol of C-peptide RIA kit (China Diagnostics Medical Corporation), briefly, the $1 \times 10^{6}$ cells/well were washed twice in DMEM (Gibco; Thermo Fisher Scientific, Inc.) containing $2 \mathrm{mM}$ glucose (Sigma-Aldrich; Merck KGaA) and cultured in this medium for $1 \mathrm{~h}$. The medium was then replaced by $2 \mathrm{ml}$ DMEM containing glucose $(0,1,5,25 \mathrm{mM}$, respectively, for each well) or $\mathrm{KCl}(0,10,20,40 \mathrm{mM}$, respectively, for each well; Sigma-Aldrich) or tolbutamide $(0,50,100 \mathrm{mM}$, respectively, for each well; Sigma-Aldrich) for $60 \mathrm{~min}$. The culture supernatants were collected after $12 \mathrm{~h}$ of incubation, centrifuged to remove debris, and concentration of C-peptide was tested by human C-peptide RIA kit.

Statistical analysis. Each experiment was performed at least three times. Data are presented as the means \pm standard error. Student's t-test was used to evaluate differences between two groups. One-way analysis of variance was used to compare multiple groups. $\mathrm{P}<0.05$ was considered to indicate a statistically significant difference.

\section{Results}

With increasing passage number, endogenous miR-32 expression and WWP2 expression are negatively associated in HuAECs. The results of the RT-qPCR analysis suggested that the mRNA expression levels of WWP2 were gradually increased in the HuAECs with continued passage (Fig. 2). Northern blot analysis confirmed that with continued passage of HuAECs, the hybridization signal of endogenous miR-32 became increasingly weaker (Fig. 2). These results indicated that endogenous miR-32 expression and WWP2 expression in HuAECs were negatively associated.

Targeted binding of miR-32 to the 3'UTR of WWP2 mRNA silences its expression. Sequence alignment, using bioinformatics analysis software (microRNA.org - Targets and Expression tools, http://34.236.212.39/microrna/home. do), identified a complementary binding site for mature miR-32 in the 3'UTR (between 1777 and $1783 \mathrm{bp}$ ) of WWP2 mRNA (Fig. 2). Luciferase reporter assays indicated that following overexpression of miR-32 in the NIH-3T3 cell line, the luciferase activity was significantly decreased when cells were cotransfected with WWP2 containing WT 3'UTR (Fig. 2). These results suggested that miR-32 could silence the mRNA expression of WWP2.

Exogenous overexpression of miR-32 effectively inhibits WWP2 expression in HuAECs. miR-32 was overexpressed in one group of HuAEC cells following infection with a lentivirus, whereas the negative control group of HuAECs were infected with miR-Mut. The two groups were cultured under the same conditions and passaged five consecutive times. Subsequently, RT-qPCR analysis indicated that, in HuAECs infected with miR-32, the mRNA expression levels of WWP2 were reduced at different passage numbers (passages 1, 2, 3, 4 and 5) compared with in the HuAECs infected with miR-Mut (Fig. 3). Furthermore, western blotting confirmed that at the 5th passage, miR-32-infected HuAECs exhibited a reduced abundance of WWP2 protein expression compared with in the miR-Mut-infected HuAECs (Fig. 3).

Exogenous overexpression of $m i R-32$ inhibits Oct4 ubiquitination by WWP 2 in HuAECs. RT-qPCR demonstrated that, at the 5th passage, miR-32-infected HuAECs exhibited significantly increased expression levels of mRNAs encoding embryonic stem cell markers (Oct4, Sox 2 and Nanog) compared with in the miR-Mut-infected HuAECs (Fig. 4). Furthermore, western blotting confirmed that, at the 5 th passage, miR-32-infected HuAECs exhibited significantly increased protein expression of embryonic stem cell markers (Oct4, Sox2 and Nanog) compared with in the miR-Mut-infected HuAECs (Fig. 4). In addition, western blotting of the co-IP samples indicated that, at the 5th passage, in miR-32-infected HuAECs Oct4 protein ubiquitination was reduced compared with in miR-Mut-infected HuAECs (Fig. 4). Immunofluorescence analysis revealed that the expression levels of Oct4, Sox2, Nanog and WWP2 were consistent with the western blotting 

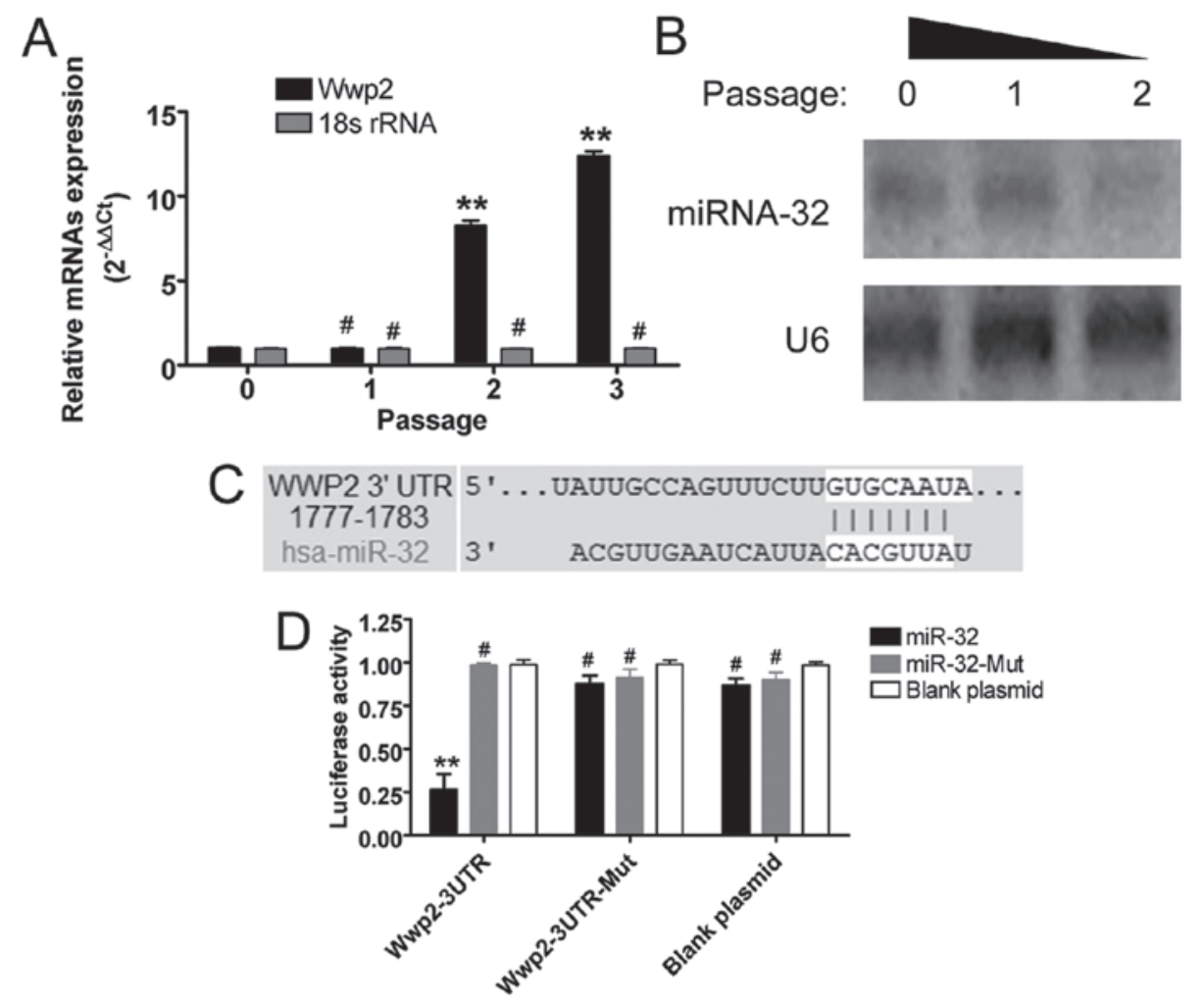

Figure 2. Expression of miR-32 and WWP2 in HuAECs and target validation. (A) Reverse transcription-quantitative polymerase chain reaction indicated that the expression levels of endogenous WWP 2 were gradually increased in HuAECs with passage ${ }^{* *} \mathrm{P}<0.01$ vs. passage 0 ; ${ }^{*} \mathrm{P}>0.05$ vs. passage 0 ; one-way ANOVA, $n=3$ ). (B) Northern blotting demonstrated that, with increasing passage of HuAECs, the hybridization signal of endogenous miR-32 became increasingly weaker. (C) Sequence alignment identified a complementary binding site for mature miR-32 in the 3'UTR of WWP2 mRNA (between 1777 and $1783 \mathrm{bp}$ ). (D) Luciferase reporter assays demonstrated that following overexpression of miR-32 in NIH-3T3 cells containing the wild-type 3'UTR of WWP2, luciferase activity was significantly decreased ( ${ }^{* *} \mathrm{P}<0.01$ vs. blank plasmid; " $\mathrm{P}>0.05$ vs. blank plasmid; one-way ANOVA, $\left.\mathrm{n}=3\right)$. $3^{\prime} \mathrm{UTR}$, 3' untranslated region; ANOVA, analysis of variance; HuAECs, human amniotic epithelial stem cells; miR/miR-32, microRNA-32; Mut, mutated; WWP, WW domain containing E3 ubiquitin protein ligase 2.

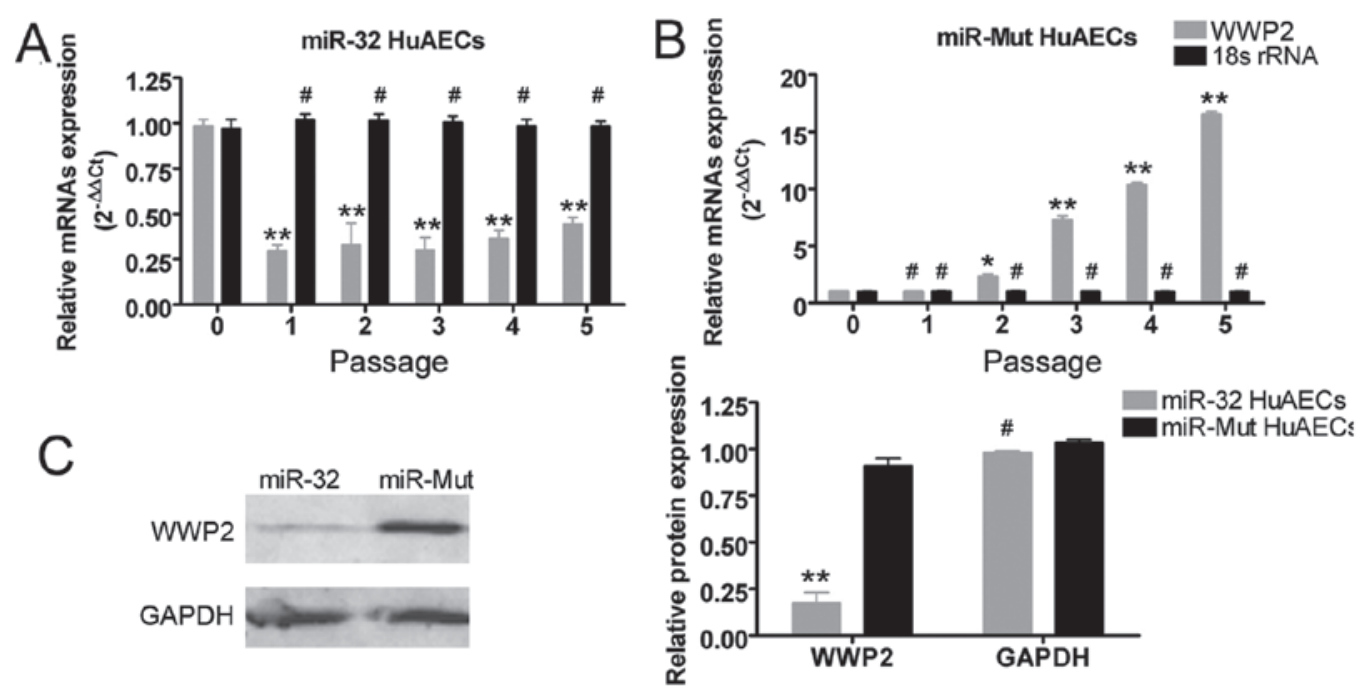

Figure 3. miR-32 inhibits WWP2 expression. (A and B) Reverse transcription-quantitative polymerase chain reaction demonstrated that, at different passages, the mRNA expression levels of WWP2 were reduced in miR-32-infected HuAECs compared with in miR-Mut-infected HuAECs $\left({ }^{* *} \mathrm{P}<0.01\right.$ vs. passage 0 ; ${ }^{*} \mathrm{P}<0.05$ vs. passage $0 ;{ }^{*} \mathrm{P}>0.05$ vs. passage 0 ; one-way analysis of variance, $\mathrm{n}=3$ ). (C) Western blot analysis confirmed, that at the 5 th passage, the protein expression levels of WWP2 were reduced in miR-32-infected HuAECs compared with in miR-Mut-infected HuAECs $\left({ }^{* *} \mathrm{P}<0.01\right.$ vs. miR-Mut HuAECs; ${ }^{*} \mathrm{P}>0.05$ vs. miR-Mut HuAECs; Student's t-test, n=3). HuAECs, human amniotic epithelial stem cells; miR-32, microRNA-32; Mut, mutated; WWP, WW domain containing E3 ubiquitin protein ligase 2.

results (Fig. 4). These findings suggested that exogenous overexpression of miR-32 effectively inhibited Oct4 ubiquitination by WWP2 in HuAECs, and maintained the expression of transcription factors associated with ESC pluripotency. 


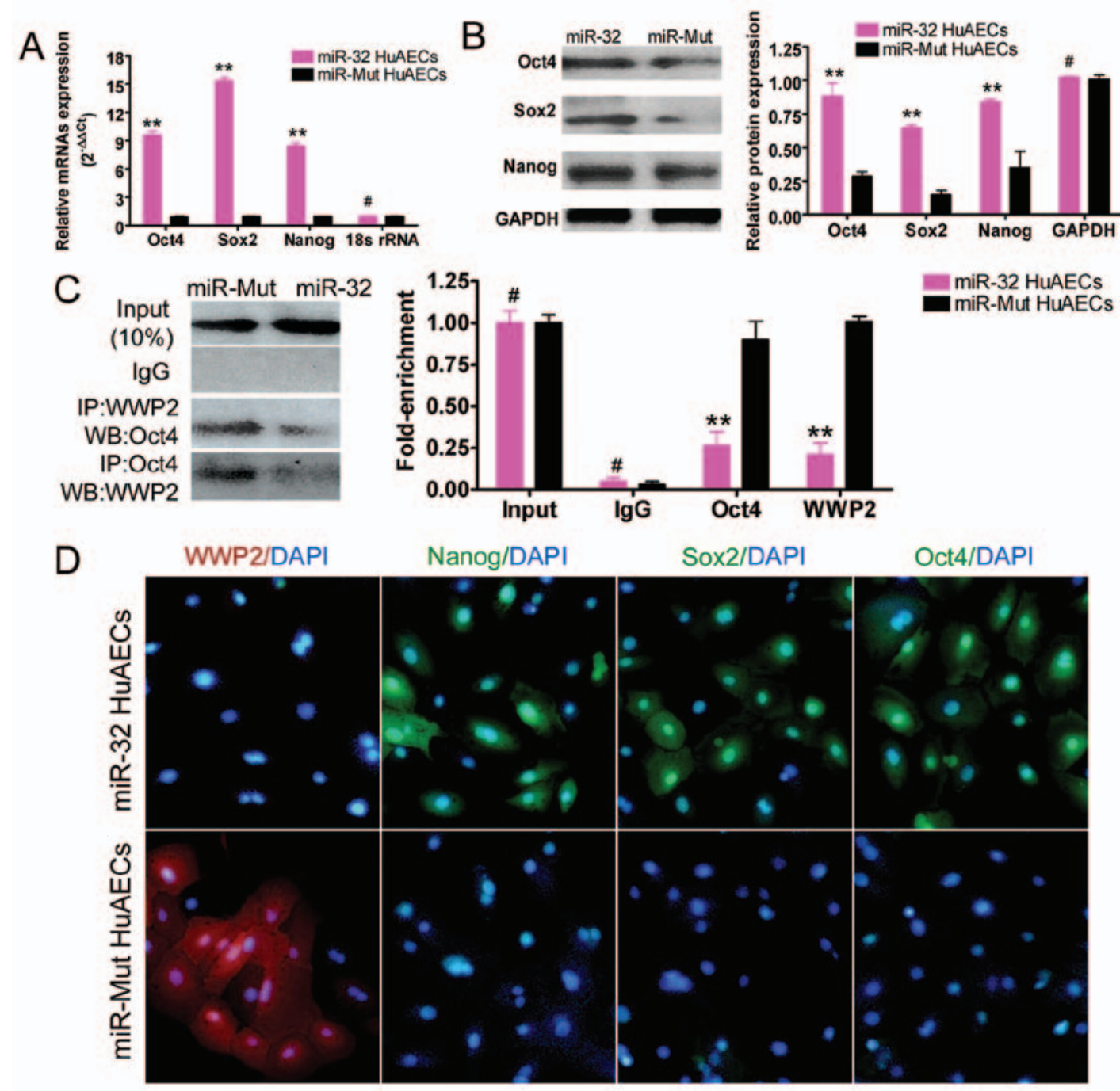

Figure 4. miR-32 effectively inhibits ubiquitination of Oct4 by WWP2. (A) Reverse transcription-quantitative polymerase chain reaction indicated that the mRNA expression levels of embryonic stem cell markers were increased in miR-32-infected HuAECs compared with in miR-Mut-infected HuAECs ( ${ }^{* *} \mathrm{P}<0.01$ vs. miR-Mut HuAECs; ${ }^{*} \mathrm{P}>0.05$ vs. miR-Mut HuAECs; Student's t-test, $\mathrm{n}=3$ ). (B) Western blot analysis confirmed that the protein expression levels of embryonic stem cell markers were increased in miR-32-infected HuAECs compared with in miR-Mut-infected HuAECs $\left({ }^{* * *} \mathrm{P}<0.01\right.$ vs. miR-Mut HuAECs; ${ }^{\#} \mathrm{P}>0.05$ vs. miR-Mut HuAECs; Student's t-test, $\mathrm{n}=3$ ). (C) Co-immunoprecipitation western blotting results indicated that ubiquitination of Oct4 protein was significantly reduced in miR-32-infected HuAECs compared with in miR-Mut-infected HuAECs $\left({ }^{* *} \mathrm{P}<0.01\right.$ vs. miR-Mut HuAECs; ${ }^{*} \mathrm{P}>0.05$ vs. miR-Mut HuAECs; Student's t-test, n=3). (D) Immunofluorescence staining demonstrated that the expression of embryonic stem cell markers in miR-32-infected HuAECs was significantly increased compared with in miR-Mut-infected HuAECs, whereas WWP2 expression was decreased (magnification, x200). DAPI, 4',6-diamidino-2-phenylindole; HuAECs, human amniotic epithelial stem cells; IgG, immunoglobulin G; IP, immunoprecipitation; miR-32, microRNA-32; Mut, mutated; Oct4, octamer-binding protein 4; Sox2, SRY-box 2; WB, western blotting; WWP, WW domain containing E3 ubiquitin protein ligase 2.

Exogenous overexpression of miR-32 effectively maintains the capability of HuAECs to differentiate into $\beta$ islet-like cells and express $\beta$ islet-like cell markers. Under specific cell culture conditions, at the 5th passage, the two HuAEC cultures (infected with miR-32 or miR-Mut) were simultaneously induced to differentiate into $\beta$ islet-like cells in vitro. After 21 days of induction, the results of RT-qPCR suggested that, in the induced cell cultures, the mRNA expression levels of $\beta$ islet-like cell biomarkers [insulin, pancreatic duodenal homeobox-1 (Pdx1), ISL LIM homeobox 1, paired box 6, NK2 homeobox 2, glucose transporter 2 and glucokinase) were significantly increased in miR-32-infected HuAECs compared with in miR-Mut-infected HuAECs (Fig. 5). Western blotting confirmed that, after induction, the protein expression levels of insulin and PDX1 were significantly increased in miR-32-infected HuAECs compared with in miR-Mut-infected
HuAECs (Fig. 5). These results suggested that exogenous overexpression of miR-32 may effectively maintain the ability of HuAECs to differentiate into $\beta$ islet-like cells and to express $\beta$ islet-like cell markers.

Exogenous overexpression of miR-32 effectively enhances $C$-peptide secretion from $\beta$ islet-like cells derived from $H u A E C s$. C-peptide is secreted into the cell culture medium in amounts equal to those of insulin secreted by the pancreas; therefore, the C-peptide content of the supernatant of cell culture medium was determined, in order to investigate the synthetic insulin-secreting capability of $\beta$ islet-like cells derived from the two HuAEC groups. Firstly, after directed induction of differentiation of HuAECs into $\beta$ islet-like cells, the levels of $\mathrm{C}$-peptide secreted by the two cell groups were analyzed at different time-points (1, 2 and 3 weeks). The 
Table III. C-peptide release.

\begin{tabular}{lcc}
\hline Variable & $\begin{array}{c}\text { miR-32 HuAECs } \\
(\mathrm{pM})\end{array}$ & $\begin{array}{c}\text { miR-Mut HuAECs } \\
(\mathrm{pM})\end{array}$ \\
\hline $\begin{array}{l}\text { Induced time } \\
\text { (weeks) }\end{array}$ & & \\
0 & & \\
1 & $0.445 \pm 0.215$ & $0.270 \pm 0.270$ \\
2 & $18.285 \pm 4.995^{\mathrm{a}}$ & $1.335 \pm 1.225$ \\
3 & $48.505 \pm 4.065^{\mathrm{a}}$ & $1.950 \pm 0.930$ \\
Glucose & $89.475 \pm 9.285^{\mathrm{a}}$ & $1.370 \pm 0.490$ \\
concentration (mM) & & \\
0 & $8.400 \pm 2.150$ & $8.385 \pm 2.905$ \\
1 & $16.650 \pm 4.110^{\mathrm{b}}$ & $9.225 \pm 1.065$ \\
5 & $47.015 \pm 6.825^{\mathrm{a}}$ & $10.375 \pm 1.705$ \\
25 & $78.865 \pm 7.375^{\mathrm{a}}$ & $12.065 \pm 1.485$ \\
KCl concentration & & \\
(mM) & & \\
0 & $1.515 \pm 0.495$ & $1.590 \pm 0.400$ \\
10 & $2.455 \pm 0.405^{\mathrm{b}}$ & $1.315 \pm 0.565$ \\
20 & $5.245 \pm 1.235^{\mathrm{a}}$ & $1.640 \pm 0.390$ \\
40 & $9.920 \pm 0.670^{\mathrm{a}}$ & $2.035 \pm 0.155$ \\
Tolbutamide & & \\
concentration (mM) & & \\
0 & $2.595 \pm 0.515$ & $3.005 \pm 0.065$ \\
50 & $4.860 \pm 0.690^{\mathrm{b}}$ & $3.580 \pm 0.590$ \\
100 & $8.990 \pm 0.970^{\mathrm{a}}$ & $2.590 \pm 0.520$ \\
\hline
\end{tabular}

HuAECs, human amniotic epithelial stem cells; miR-32, microRNA-32. ${ }^{\mathrm{a}} \mathrm{P}<0.01$ vs. miR-Mut HuAECs; ${ }^{\mathrm{b}} \mathrm{P}<0.05$ vs. miR-Mut HuAECs.

results demonstrated that, with increasing time after induction, the levels of C-peptide secreted by miR-32-infected HuAECs were much greater compared with those secreted by miR-Mut-infected HuAECs (Fig. 6 and Table III). Secondly, the levels of C-peptide secreted following stimulation of $\beta$ islet-like cells with various concentrations of glucose were compared. The results indicated that with increasing glucose concentration, the amount of C-peptide secreted by $\beta$ islet-like cells derived from miR-32-infected HuAECs was significantly increased compared with that secreted by cells derived from miR-Mut-infected HuAECs (Fig. 6 and Table III). In addition, following supplementation of the culture medium with various concentrations of $\mathrm{KCl}$, the results indicated that, with increasing $\mathrm{KCl}$ concentration, the amount of C-peptide secreted by $\beta$ islet-like cells derived from miR-32-infected HuAECs was much greater than that secreted by cells derived from miR-Mut-infected HuAECs (Fig. 6 and Table III). Furthermore, since blockage of the functionally sensitive intracellular $\mathrm{K}_{\text {АTP }}$ ion channels may induce an increase in C-peptide secretion, tolbutamide, an ATP-sensitive $\mathrm{K}_{\text {АTP }}$ ion channel inhibitor, was added to the culture medium of each cell group. The results indicated that with increasing tolbutamide concentration, $\mathrm{C}$-peptide secretion of $\beta$ islet-like cells derived from miR-32-infected HuAECs was much higher than that of cells derived from miR-Mut-infected

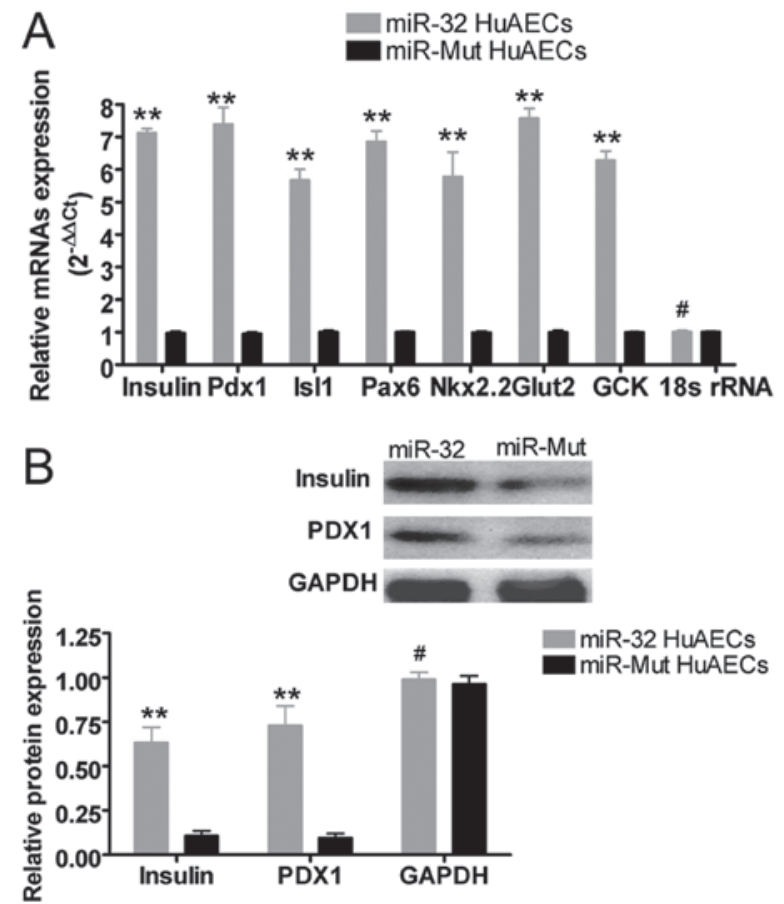

Figure 5. miR-32 maintains the ability of HuAECs to differentiate into $\beta$ islet-like cells. (A) Reverse transcription-quantitative polymerase chain reaction analysis suggested that, after induction, the mRNA expression levels of $\beta$ islet-like cell biomarkers were increased in miR-32-infected HuAECs compared with in miR-Mut-infected HuAECs $\left({ }^{* *} \mathrm{P}<0.01\right.$ vs. miR-Mut HuAECs; "P>0.05 vs. miR-Mut HuAECs; Student's t-test, n=3). (B) Western blot analysis demonstrated that, after induction, the protein expression levels of insulin and PDX1 were increased in miR-32-infected HuAECs compared with in miR-Mut-infected HuAECs $\left({ }^{* *} \mathrm{P}<0.01\right.$ vs. miR-Mut HuAECs; ${ }^{*} \mathrm{P}>0.05$ vs. miR-Mut HuAECs; Student's t-test, n=3). GCK, glucokinase; Glut2, glucose transporter 2; HuAECs, human amniotic epithelial stem cells; Is11, ISL LIM homeobox 1; miR-32, microRNA-32; Mut, mutated; Nkx2.2, NK2 homeobox 2; Pax1, paired box 6; Pdx1, pancreatic duodenal homeobox-1.

HuAECs (Fig. 6 and Table III). These findings confirmed that exogenous overexpression of miR-32 could effectively enhance the $\mathrm{C}$-peptide secretion of $\beta$ islet-like cells.

\section{Discussion}

HuAECs are derived from trophoblasts, but retain the characteristics of ESCs (1-3). Although HuAECs are difficult to grow in vitro (they can be grown for a maximum of five passages), they have pluripotent capabilities, which are similar to stem cells (1-3). HuAECs are able to differentiate into numerous human tissues and cells that belong to the three human germ layers, under various induction conditions (1-3). In addition, they possess specific physiological and biochemical functions of adult cells; therefore, they are considered promising candidates for cell therapy (1-3). However, it is difficult to maintain the pluripotency of HuAECs in vitro (1-3). The present study demonstrated that the expression levels of Oct4, WWP2 and Nanog, which are transcription factors associated with stem cell pluripotency, were markedly decreased with increasing passage number, leading directly to the loss of pluripotency of HuAECs and an inability to induce differentiation into certain adult cells. Therefore, investigating the mechanism underlying the maintenance of stem cell pluripotency may help to improve the culture efficiency of HuAECs and maintain their 'stemness'. Previous 

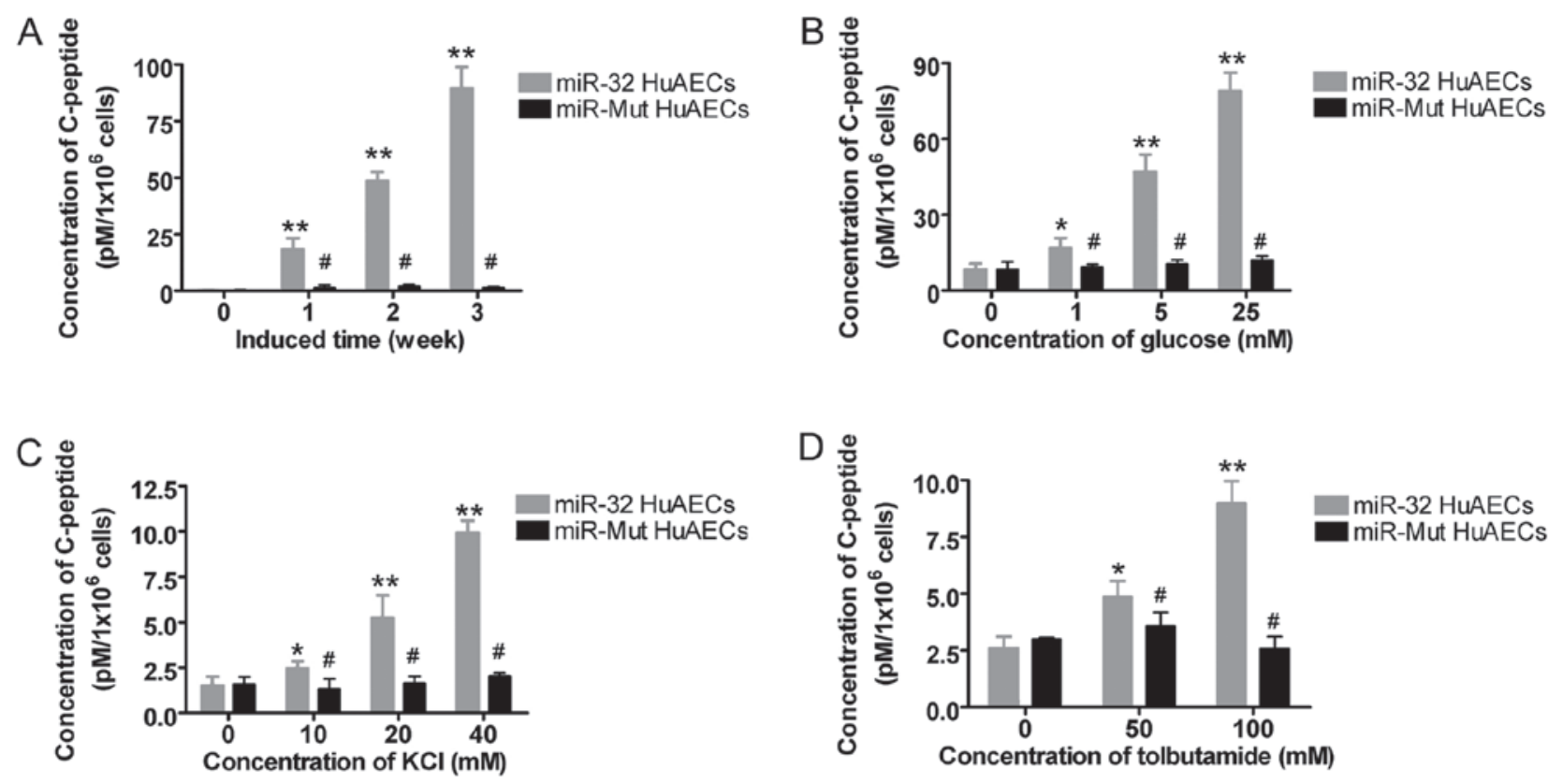

Figure 6. miR-32 enhances the secretion of C-peptide from $\beta$ islet-like cells derived from HuAECs. (A) With increasing time after induction, C-peptide secretion from $\beta$ islet-like cells derived from miR-32-infected HuAECs was significantly increased compared with that from cells derived from miR-Mut-infected HuAECs $\left({ }^{* * *} \mathrm{P}<0.01\right.$ vs. miR-Mut HuAECs at 0 week; ${ }^{\#} \mathrm{P}>0.05$ vs. miR-Mut HuAECs at 0 week; one-way ANOVA, $\left.\mathrm{n}=3\right)$. (B) With increasing glucose concentration in the medium, C-peptide secretion from $\beta$ islet-like cells derived from miR-32-infected HuAECs was significantly increased compared with that from cells derived from miR-Mut-infected HuAECs $\left({ }^{* *} \mathrm{P}<0.01\right.$ vs. $0 \mathrm{mM}$ glucose-treated miR-Mut HuAECs; ${ }^{*} \mathrm{P}<0.05$ vs. $0 \mathrm{mM}$ glucose-treated miR-Mut HuAECs; ${ }^{*} \mathrm{P}>0.05$ vs. $0 \mathrm{mM}$ glucose-treated miR-Mut HuAECs, one-way ANOVA; $\mathrm{n}=3$ ). (C) With increasing concentration of $\mathrm{KCl}$ in the medium, $\mathrm{C}$-peptide secretion from $\beta$ islet-like cells derived from miR-32-infected HuAECs was significantly increased compared with that from cells derived from miR-Mut-infected HuAECs ("P $<0.01$ vs. 0 mM KCl-treated miR-Mut HuAECs; "P<0.05 vs. 0 mM KCl-treated miR-Mut HuAECs; "P>0.05 vs. 0 mM KCl-treated miR-Mut HuAECs; one-way ANOVA, $\mathrm{n}=3$ ). (D) With increasing concentration of tolbutamide in the medium, C-peptide secretion from $\beta$ islet-like cells derived from miR-32-infected HuAECs was significantly increased compared with that of cells derived from miR-Mut-infected HuAECs ( ${ }^{* *} \mathrm{P}<0.01$ vs. 0 mM tolbutamine-treated miR-Mut HuAECs; ${ }^{*} \mathrm{P}<0.05$ vs. $0 \mathrm{mM}$ tolbutamine-treated miR-Mut HuAECs; ${ }^{\# P}>0.05$ vs. $0 \mathrm{mM}$ tolbutamine-treated miR-Mut HuAECs; one-way ANOVA, $\mathrm{n}=3$ ). ANOVA, analysis of variance; HuAECs, human amniotic epithelial stem cells; miR-32, microRNA-32 Mut, mutated;

studies have indicated that the transcription factors associated with pluripotent stem cells serve important regulatory roles in in vitro and in vivo proliferation, the maintenance of pluripotency, and the directed differentiation of stem cells. The present study aimed to determine why the expression level of WWP2 was slowly increased in HuAECs alongside passage number. It has previously been reported that Oct4 activity is regulated by numerous factors $(9,10,13,14)$. At the gene expression level, there are two regulatory pathways: Transcriptional modification and post-transcriptional modification. Generally, in adult cells, the Oct4 gene is inactivated, and epigenetic analyses indicated that the $\mathrm{CpG}$ islands in the gene promoter are highly methylated $(9,10,13)$. In addition, binding sites in the promoter and in histones, including H3K27 and H3K9, are modified by methylation and deacetylation, which cause direct downregulation of gene transcription, ultimately affecting gene expression $(9,10,13)$. These modifications are at the transcriptional level $(9,10,13)$. At the post-transcriptional level, some preliminary studies have suggested that endogenous Oct4 protein is degraded in ESCs following prolonged culture in vitro via the main degradation pathway of protein ubiquitination $(9,10,13,14)$. With continued passage of ESCs, WWP2 may be activated and bind to the Oct4 protein, thereby triggering subsequent ubiquitination and degradation, thus leading to loss of Oct4 protein expression and reduced pluripotency of ESCs $(9,10,13,14)$. These results suggested that, in order to maintain Oct4 expression, blocking the expression and activity of WWP2 is crucial $(9,10,13,14)$.
Based on these findings, the present study focused on the regulatory mechanism of WWP2 ubiquitin ligase in HuAECs, in order to provide a novel hypothesis regarding maintenance of the pluripotency of stem cells in vitro. The results confirmed that WWP2 is regulated by endogenous miR-32, particularly in the primary culture stage when miR-32 expression is relatively high. However, with consecutive passages of HuAECs, miR-32 expression was significantly reduced, whereas endogenous WWP2 expression was increased. HuAECs were then induced to overexpress exogenous miR-32 and were compared with HuAECs infected with miR-Mut. The results demonstrated that WWP2 expression in miR-32-infected cells was significantly decreased, whereas the expression of transcription factors associated with pluripotency (Oct4, Sox 2 and Nanog) were significantly increased, thus suggesting that miR-32 may significantly inhibit WWP2 expression and promote the expression of pluripotency-associated transcription factors. Furthermore, the results of co-IP demonstrated that, crosslinking between WWP2 and Oct4 proteins was significantly increased in miR-Mut expressed HuAECs but not in miR-32 expressed HuAECs. These findings suggested that ubiquitination of Oct 4 was enhanced, whereas miR-32 overexpression in HuAECs significantly inhibited endogenous WWP2 protein expression, and crosslinking between Oct4 and WWP2 was also decreased.

In the present study, HuAECs infected with either miR-32 or miR-Mut were induced to differentiate into $\beta$ islet-like cells. 
Molecular analyses indicated that following directed induction, the expression levels of $\beta$ islet-like cell markers were significantly increased in miR-32-infected HuAECs compared with in miR-Mut-infected HuAECs. Furthermore, with increasing induction time, C-peptide secretion was also increased from miR-32-infected HuAECs compared with in miR-Mut-infected HuAECs. In addition, C-peptide secretion of the two HuAEC groups were investigated following stimulation with various concentrations of glucose. With increasing glucose concentration in the medium, the amount of C-peptide secreted by $\beta$ islet-like cells derived from miR-32-infected HuAECs was much greater than that secreted by cells derived from miR-Mut-infected HuAECs. In addition, with increasing concentrations of $\mathrm{KCl}$ in the medium, C-peptide secretion from $\beta$ islet-like cells derived from miR-32-infected HuAECs was significantly increased compared with that from cells derived from miR-Mut-infected HuAECs. Furthermore, increasing concentrations of tolbutamide (a hypoglycemic drug) were added to the HuAEC groups, which increased C-peptide secretion from $\beta$ islet-like cells derived from miR-32-infected HuAECs compared with that secreted from $\beta$ islet-like cells derived from miR-Mut-infected HuAECs. These results confirmed that after WWP2 was silenced by miR-32 overexpression in miR-32-infected HuAECs, the responsiveness to sugar concentrations in the culture medium and C-peptide secretion from $\beta$ islet-like cells derived from them were significantly increased compared with in cells derived from miR-Mut-infected HuAECs.

In conclusion, miR-32 may prevent the ubiquitination and degradation of Oct4 by inhibiting the expression of WWP2, thus promoting the maintenance of stem cell pluripotency during consecutive passages of HuAECs in vitro.

\section{Acknowledgements}

The present study was supported by grants from the National Natural Science Foundation of China (grant nos. 81471461 and 81202811), the China Postdoctoral Science Foundation (grant nos. 2014M550250 and 2015T80455), the Shanghai Municipal Health Bureau Fund (grant nos. 14XD1403200, SHDC12013114, 20142006, PWZxq2014-02 and 20124320), the Shanghai Natural Science Foundation (grant no. 16ZR1434000), the projects sponsored by the Development Fund for Shanghai Talents (grant no. 2017054) and the Fund for Xinglin Talents of Shanghai University of TCM (grant no. 201707081).

\section{Competing interests}

The authors declare that they have no competing interests.

\section{References}

1. Liu T, Wu J, Huang Q, Hou Y, Jiang Z, Zang S and Guo L: Human amniotic epithelial cells ameliorate behavioral dysfunction and reduce infarct size in the rat middle cerebral artery occlusion model. Shock 29: 603-611, 2008.

2. Liu YH, Chan J, Vaghjiani V, Murthi P, Manuelpillai U and Toh BH: Human amniotic epithelial cells suppress relapse of corticosteroid-remitted experimental autoimmune disease. Cytotherapy 16: 535-544, 2014.
3. Liu XY, Chen J, Zhou Q, Wu J, Zhang XL, Wang L and Qin XY: In vitro tissue engineering of lamellar cornea using human amniotic epithelial cells and rabbit cornea stroma. Int J Ophthalmol 6: 425-429, 2013.

4. Verma R, Oania R, Graumann J and Deshaies RJ: Multiubiquitin chain receptors define a layer of substrate selectivity in the ubiquitin-proteasome system. Cell 118: 99-110, 2004.

5. Jennissen HP: Ubiquitin and the enigma of intracellular protein degradation. Eur J Biochem 231: 1-30, 1995.

6. Guardavaccaro D and Pagano M: Oncogenic aberrations of cullin-dependent ubiquitin ligases. Oncogene 23: 2037-2049, 2004.

7. Zhao B, Bhuripanyo K, Schneider J, Zhang K, Schindelin H, Boone D and Yin J: Specificity of the E1-E2-E3 enzymatic cascade for ubiquitin C-terminal sequences identified by phage display. ACS Chem Biol 7: 2027-2035, 2012.

8. Chen $\mathrm{Z}$ and Lu W: Roles of ubiquitination and SUMOylation on prostate cancer: Mechanisms and clinical implications. Int J Mol Sci 16: 4560-4580, 2015.

9. Liao B and Jin Y: Wwp2 mediates Oct4 ubiquitination and its own auto-ubiquitination in a dosage-dependent manner. Cell Res 20: 332-344, 2010.

10. Xu H, Wang W, Li C, Yu H, Yang A, Wang B and Jin Y: WWP2 promotes degradation of transcription factor OCT4 in human embryonic stem cells. Cell Res 19: 561-573, 2009.

11. Chen W, Jiang X and Luo Z: WWP2: A multifunctional ubiquitin ligase gene. Pathol Oncol Res 20: 799-803, 2014.

12. Zou W, Chen X, Shim JH, Huang Z, Brady N, Hu D, Drapp R, Sigrist K, Glimcher LH and Jones D: The E3 ubiquitin ligase Wwp2 regulates craniofacial development through mono-ubiquitylation of Goosecoid. Nat Cell Biol 13: 59-65, 2011.

13. Xu HM, Liao B, Zhang QJ, Wang BB, Li H, Zhong XM, Sheng HZ, Zhao YX, Zhao YM and Jin Y: Wwp2, an E3 ubiquitin ligase that targets transcription factor Oct-4 for ubiquitination. J Biol Chem 279: 23495-23503, 2004.

14. Li H, Zhang Z, Wang B, Zhang J, Zhao Y and Jin Y: Wwp2-mediated ubiquitination of the RNA polymerase II large subunit in mouse embryonic pluripotent stem cells. Mol Cell Biol 27: 5296-5305, 2007.

15. Liu T, Chen Q, Huang Y, Huang Q, Jiang L and Guo L: Low microRNA-199a expression in human amniotic epithelial cell feeder layers maintains human-induced pluripotent stem cell pluripotency via increased leukemia inhibitory factor expression. Acta Biochim Biophys Sin (Shanghai) 44: 197-206, 2012.

16. Liu T, Cheng W, Huang Y, Huang Q, Jiang L and Guo L: Human amniotic epithelial cell feeder layers maintain human iPS cell pluripotency via inhibited endogenous microRNA-145 and increased Sox 2 expression. Exp Cell Res 318: 424-434, 2012.

17. Liu T, Cheng W, Liu T, Guo L, Huang Q, Jiang L, Du X, $\mathrm{Xu} F$, Liu Z and Lai D: Human amniotic epithelial cell feeder layers maintain mouse embryonic stem cell pluripotency via epigenetic regulation of the c-Myc promoter. Acta Biochim Biophys Sin (Shanghai) 42: 109-115, 2010.

18. Zou G, Liu T, Zhang L, Liu Y, Li M, Du X, Xu F, Guo L and Liu Z: Induction of pancreatic $\beta$-cell-like cells from $\mathrm{CD} 44^{+/} \mathrm{CD} 105^{+}$human amniotic fluids via epigenetic regulation of the pancreatic and duodenal homeobox factor 1 promoter. DNA Cell Biol 30: 739-748, 2011.

19. Livak KJ and Schmittgen TD: Analysis of relative gene expression data using real-time quantitative PCR and the 2(-Delta Delta C(T)) Method. Methods 25: 402-408, 2001.

20. Liu T, Qin W, Hou L and Huang Y: MicroRNA-17 promotes normal ovarian cancer cells to cancer stem cells development via suppression of the LKB1-p53-p21/WAF1 pathway. Tumour Biol 36: 1881-1893, 2015.

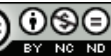

This work is licensed under a Creative Commons Attribution-NonCommercial-NoDerivatives 4.0 International (CC BY-NC-ND 4.0) License. 